\title{
El movimiento de la medicina basada en la evidencia: Alcances conceptuales y teóricos
}

\author{
Paula Bedregal G ${ }^{1}$, Carlos Cornejo A ${ }^{2}$. \\ Evidence based medicine: \\ Theoretical and conceptual issues
}

Evidence based decision making in medicine is, nowadays, a movement with political and practical implications. This article performs a historical description of evidence based medicine and its underlying conceptual and philosophical premises. An open dialogue to recognize the diverse sources of evidence that modify clinical practice is proposed, to improve decision making in this field (Rev Méd Chile 2005; 133: 977-82).

(Key-words: Evidence-Based Medicine; Decision making; Philosophy, medical)

Recibido el 20 de enero, 2005. Aceptado el 13 de junio, 2005.

${ }^{1}$ Departamento de Salud Pública, Escuela de Medicina, Pontificia Universidad Católica de Chile. ${ }^{2}$ Escuela de Psicología, Pontificia Universidad Católica de Chile. Santiago de Chile

$\mathrm{D}$ urante las últimas décadas ha surgido con fuerza un movimiento en medicina, que enfatiza la importancia de basar la toma de decisiones médicas en «evidencia». Así, la Medicina Basada en la Evidencia (MBE) ha sido reconocida como una de las ideas más influyentes durante $2001^{1}$. Afirmaciones centrales de la MBE son, que existe un cúmulo de evidencias científicas que deben ser la base de la práctica clínica y que las prácticas clínicas y en poblaciones deben someterse al análisis científico ${ }^{2}$.

Este movimiento también ha influido en el ámbito político a través de las llamadas spolíticas basadas en evidencia». Sin embargo, Friedman ${ }^{4}$ sugiere que para la práctica política lo relevante

Correspondencia a: Dra. Paula Bedregal G. Dirección: Marcoleta 434, Santiago Centro. Fono: 686 3038. Fax: 633 1840. E mail: pbedrega@med.puc.cl no es que las políticas estén \asadas en ciencias» sino más bien que las decisiones políticas consideren la información científica de alta calidad, ya que se basan en un conjunto de conocimientos científicos y no científicos.

Dados el éxito de este movimiento y sus visibles repercusiones en la toma de decisiones clínicas y de políticas públicas, resulta importante pensar en los fundamentos teóricos que sostienen a la MBE. Para esto, realizaremos una breve descripción histórica del movimiento en la medicina, analizaremos el problema conceptual de גa evidencia»y algunos presupuestos filosóficos subyacentes.

Orígenes históricos de la Medicina Basada en Evidencia. La MBE tuvo sus orígenes a mediados del siglo XIX, en París. En aquel entonces, se producía un interesante debate cultural entre los defensores de la medicina como arte y la medici- 
na como ciencia. Por ejemplo, el médico francés Risueño d'Amador entendía al médico como un artista, que debía confiar en su intuición y en su «sentimiento» médico al atender a un paciente ${ }^{5}$. Por el contrario, los propulsores de la medicina como ciencia rechazaban esa idea y defendían la necesidad de practicar la medicina en función de hechos. Los hechos aparecen después de realizar observaciones exactas de los tratamientos, definir las enfermedades, conocer su historia natural y los efectos de los tratamientos ${ }^{6}$. El método defendido era el de las llamadas 'ciencias empíricas', fundado en la observación y descripción de un objeto de conocimiento (en este caso, la enfermedad y no el enfermo), el cual es pensado como ajeno al observador (en este caso, el médico), y sobre el cual este último debe emitir un juicio racional y neutral. Por lo tanto, se sostenía que el médico debía ser un científico neutral con habilidades clínicas. Estos presupuestos quedaron en la base de la formación médica, incorporándose formalmente las ciencias básicas al curriculum médico en la primera mitad del siglo XX.

El concepto de MBE se originó en la Universidad de McMaster, Canadá, a comienzos de la década 1990-997. Años antes, Cochrane ${ }^{8}$ publicó un ensayo, señalando que el sistema de salud inglés se encontraba en una encrucijada: incremento en los gastos para mínimos resultados sanitarios. Cochrane sugirió que la variabilidad de la práctica clínica y la inflación, podrían ser controladas por la «ciencia», en particular por el uso de los ensayos clínicos controlados (ECC).

El interés por los estudios experimentales, en particular los ECC, se explica por el desarrollo que este tipo de diseños ha tenido durante el siglo XX. En 1917, se introdujeron las primeras experiencias con técnicas de doble ciego; en 1948 se realizaron los primeros estudios en medicina con aleatorización de sujetos. Después del desastre de la talidomida, en 1961, durante 1963 se formalizó en los Estados Unidos el uso de ECC para evaluar fármacos, antes de hacerlos disponibles para su uso en población e incorporarlos al registro nacional de drogas (que data de 1950) ${ }^{9}$.

En política pública, el movimiento de MBE fue recogido en el Reino Unido a raíz de un informe del House of Lords Select Committe on Science and Technology (1988), que planteó la necesidad de readecuar el sistema de salud inglés. Así se generó en 1991 la estrategia nacional inglesa de investigación y desarrollo, cuyo objetivo es obtener de la investigación una información relevante para las políticas de salud, la práctica clínica y la toma de decisiones para la gestión de servicios ${ }^{10}$. Este modelo ha sido seguido por muchos países, incluido Chile ${ }^{11}$.

¿Qué cuenta como 'evidencia'? Los creadores del concepto de MBE, la definen como el suso conciente, explícito y juicioso de la mejor evidencia disponible para tomar decisiones respecto de la atención individual de los pacientes ${ }^{2}$. Para ellos, la práctica de una MBE implica integrar la experiencia clínica junto con la evidencia externa que proviene de la investigación sistemática. De esta definición -ampliamente aceptada- se desprende la prescripción de cómo debe realizarse la toma de decisiones médicas: con «a mejor evidencia disponible». Lo que, sin embargo, no quedó explícito, es qué cuenta como «evidencia» y qué no. Porque en principio, nada es «evidencia»en sí mismo, con independencia del objetivo perseguido y del criterio que opere para evaluar los hechos observados.

Existe, por lo tanto, una conexión filosófica íntima entre el debate médico actual sobre la importancia de la 'evidencia' y el antiguo debate epistemológico sobre qué prueba la validez de un determinado conocimiento o de una determinada afirmación.

Desde tempranas épocas, el asunto de la evidencia ha sido un tema de debate en filosofía del conocimiento. Así, en la filosofía griega se conoció y divulgó la oposición terminológica entre la episteme y la doxa. La episteme correspondía al conocimiento certero, trascendente y verdadero, al cual deberían tender la filosofía y la ciencia. La doxa, por otra parte, correspondía a una pieza de conocimiento -supongamos, una afirmación- con validez relativa o circunscrita al ámbito de las opiniones subjetivas. El gran problema de la antigua epistemología era, entonces, estudiar los modos por los cuales la doxa llega a ser episteme, la opinión subjetiva se convierte en conocimiento verdadero, es decir, en una creencia cuya verdad ha sido justificada.

Durante la historia de la filosofía del conocimiento, han surgido múltiples respuestas sobre el modo válido de acceso a la episteme. En general 
se distinguen dos grandes aproximaciones filosóficas: el fundacionalismo y el coherentismo.

La primera compara al conocimiento verdadero con una estructura, en cuyas bases es posible develar y explicitar ciertas creencias fundantes. Las distintas teorías fundacionalistas representaban diferentes posiciones sobre cuáles creencias básicas cuentan como fundantes y cuáles no. Una de las más conocidas -la teoría del empirismo lógico- considera que el piso fundacional de cualquier episteme lo constituye la correspondencia entre el mundo y la afirmación. Es decir, toda episteme debería ser capaz de reducirse a un conjunto finito de afirmaciones, cada una ellas susceptible de ser verificada empíricamente en el mundo.

Por otra parte, las filosofías coherentistas rechazan la idea de que existan elementos fundantes externos a las teorías mismas y, por lo tanto, más bien apelan a la relación entre las creencias. La idea es que un conjunto de creencias son coherentes unas con otras o no lo son. Así, una teoría científica sería conocimiento certero si define un conjunto de creencias coherentes entre sí. Ambas posiciones tienen sus debilidades y son parte de la discusión contemporánea sobre qué constituye ciencia ${ }^{13}$.

En la prescripción teórica de la MBE puede reconocerse ecos de debates filosóficos antiguos y, además, una clara preferencia epistemológica fundacionalista, que va a otorgar importancia central a la 'evidencia', entendida como los datos empíricos accesibles mediante las metodologías usuales disponibles y consideradas legítimas por la institución social llamada 'ciencia'.

¿Es evidente qué es 'evidencia'? Hemos tratado de mostrar que la evidencia buscada por la MBE no es cualquier evidencia, sino aquella que satisface los criterios de ser empírica científicamente. Sin embargo, no es claro que ésa sea precisamente la definición de 'evidencia' que prevalece en la comunidad de usuarios del concepto: médicos y equipo de salud. Una exploración de la literatura científica publicada nos muestra, sorprendentemente, que el concepto dista de ser unívoco y, adicionalmente, que progresivamente se ha transformado en un concepto más normativo que descriptivo.

Dos estudios realizados sobre MBE en situaciones culturales diferentes, muestran que el con- cepto «evidencia» es ambiguo. En varios estudios de casos ingleses, se reporta que los clínicos, especialistas y no especialistas, consideran que la evidencia se construye localmente y que la $\mathrm{MBE}$ corresponde a un conocimiento formal explícito, que no necesariamente se relaciona y resuelve las preguntas de la clínica ${ }^{10}$. Se argumenta que, hasta ahora, no se ha observado que contar con «buena» evidencia lleve a resultados clínicos y epidemiológicos exitosos. De esta manera, para los clínicos entrevistados, esta evidencia científica no es una fuente absoluta ni transparente, ya que se construye sobre debates y controversias.

En Chile ${ }^{14}$, un estudio en atención primaria de salud mostró nuevamente el carácter polisémico del concepto. Para médicos implicados en políticas sectoriales, cuenta como evidencia científica aquella que permite lograr control social. Para los administradores que están a cargo del presupuesto, es evidencia científica aquella que permite controlar costos. Para médicos que trabajan en atención primaria, lo que cuenta como evidencia es la experiencia clínica. Los otros profesionales de la salud aducen falta de tiempo para manejar las evidencias científicas, porque en la práctica lo que cuenta como evidencia para su ejercicio profesional es el contexto de trabajo y el paciente. En general, para los entrevistados el proceso político y la relación médico-paciente son más importantes que el conocimiento científico propugnado por la MBE.

En ambos estudios queda claro que el concepto de 'evidencia' posee una fuerza normativa y moral que excede su aparente origen científico. En filosofía se denomina 'criptonormativos' a aquellos conceptos -como el de MBE- que contienen no sólo un aspecto descriptivo científico, sino que conllevan una carga valórica adicional, en este caso, positiva. En este sentido, Valkenburg y cols $^{15}$ concluyeron que es necesario negociar la definición del concepto y reducir su carga moral, por las consecuencias sociales que esto tiene en la clínica y en la gestión de recursos.

En síntesis, no es evidente qué es evidencia. La concepción de 'evidencia' de la formulación original de la teoría de la MBE -que, como hemos visto, es criptonormativa, fundacionalista y empirista- llega a los servicios asistenciales, pero es modificada sustancialmente según el actor. Es interesante que, parece suscitarse una negocia- 
ción, en que diversas concepciones científicas y formas de conocer, son tomadas en consideración simultáneamente para realizar un juicio clínico. Cuán basado esté en ensayos clínicos 'científicos' o no dependerá de la posición que cada clínico tenga respecto de estas suposiciones, muchos de ellos de carácter inconsciente.

Algunos supuestos filosóficos del movimiento de la MBE. La MBE nace bajo el supuesto de su carácter a-teórico. Es decir, aparentemente no está amparada en ninguna filosofía de la ciencia y parece estar basada exclusivamente en hechos prácticos. En este sentido, se afirma que es pragmática. Sin embargo, es consensual en teoría de la ciencia, que aquella pretensión de a-teoricidad no es posible. Muchos autores, entre otros, Popper ${ }^{16}, \mathrm{Kuhn}^{17} \mathrm{y}$ Feyerabend ${ }^{18}$ han argumentado y demostrado con datos lógicos e historiográficos que es imposible pretender argumentar desde un punto de vista privilegiado, objetivo en forma absoluta y limpio de toda preconcepción. La observación de un dato empírico 'puro' -la llamada observación 'from God's eyes' o 'the vision from nowhere'- es un imposible. «os científicos (y los clínicos) no son individuos que observan el mundo a partir de cero; son participantes de un universo cultural y lingüístico en el que se insertan sus proyectos individuales y colectivos ${ }^{19}$. Quine ${ }^{20}$, uno de los filósofos más importantes del siglo XX, advirtió que no hay observación pura, como tampoco hay teoría pura sin correlato empírico: «or eso son paralelas las consecuencias. Del mismo modo que sólo podemos hablar significativamente de la verdad de una sentencia dentro de alguna teoría o esquema conceptual, así tampoco podemos hablar significativamente de sinonimia interlingüística más que dentro del discurso de algún concreto sistema de hipótesis analíticas».

Esta pretensión de a-teoricidad parece del todo inapropiada ya que, como hemos argumentado, al examinar sus suposiciones o principios es posible notar que existe un abordaje de la «evidencia científica»desde una perspectiva empirista clásica, en la que se defiende la necesidad de rigor, replicabilidad e independencia del investigador respecto de lo observado.

La MBE también se puede asociar al Positivismo Lógico, en su afán verificacionista. Se busca la evidencia empírica, con el menor sesgo de inter- pretación posible, respecto de una pregunta clínica para verificar un hecho. La mejor evidencia es aquella verificable y ajustada a teorías axiomáticas. En este sentido el conocimiento científico es una creencia verdadera justificada a partir de sus estructuras fundantes.

En medicina, esto no es sorprendente, dada la tendencia a valorar el modelo de las ciencias biológicas, químicas y físicas. Pese a esto, el debate contemporáneo pone de manifiesto que este abordaje, enfocado en la enfermedad y no en el enfermo, se contradice con los actuales postulados sobre el proceso salud-enfermedad, la visión integrada sobre los determinantes de la enfermedad en las personas ${ }^{21}$, la necesidad de transferir poder a los usuarios de los sistemas de salud ${ }^{22} \mathrm{y}$ volver hacia la persona enferma ${ }^{23}$.

Otro punto ciego teórico de la MBE es su valoración (no científicamente sustentable) de la información abstracta por sobre la información contextualizada. Cualquier afirmación contextualmente relevante o proveniente del sentido común corre el severo riesgo de ser descartada a priori si no ha sido legitimada a través de una metodología experimental o estadística. La relevancia de la evidencia empírica documentada con los procedimientos estándares de las ciencias básicas, por sobre la evidencia como posibilidad y sentido (común) adquiere muchas veces el carácter de culto de la afirmación científica, que por definición siempre es potencialmente falible. En la práctica ello se traduce en la importancia otorgada a la metódica del ECC.

Los diseños cualitativos se han incluido últimamente entre las evidencias científicas, asignándoseles usualmente una tácita valoración inferior. Equivale a asignar menor valor a la pregunta por el sentido y el significado, que aquella por el hecho empírico. En la práctica clínica ello es un error, pues implícitamente se estaría despojando al objeto de estudio y al sujeto (investigador) de una de sus características constitutivas, a saber, su lenguaje y significado. Para la medicina, esto significa una vuelta atrás en la comprensión teórica del proceso salud-enfermedad y del enfermo. Una integración metodológica parecería más razonable.

Es importante, no obstante, subrayar que el gran aporte de la MBE ha sido interesar a los clínicos y otros en evaluar la calidad de la 
investigación científica médica publicada, y estimular la búsqueda de parámetros de calidad para la gestión clínica.

Y ahora... ¿hacia dónde? Nos encontramos ante un tiempo de cambio en la medicina. Cambio que se suscita en un entorno determinado por las regulaciones impuestas desde otras profesiones (la economía y el derecho), en que se proclama la relevancia del cliente (el paciente como consumidor de asistencia sanitaria).

Observamos un cambio en la filosofía de la ciencia y en la práctica científica. Como señala Prigogine ${ }^{24}$, estamos llegando al final de la ciencia convencional, de la ciencia determinista, lineal, homogénea y presenciamos el surgimiento de una conciencia de discontinuidad, de no linealidad, de la diferencia y de la necesidad de diálogo. Dos alternativas han emergido con fuerza, en un contexto en que la genética y las neurociencias plantean retos en la comprensión del hombre. Por una parte el modelo de Reducción Teórica (una ciencia única, con métodos únicos, lenguaje único) propuesta como un vehículo para vincular el trabajo de diferentes disciplinas, que no ha sido del todo exitoso. Ello porque no siempre es esperable que las propiedades que descubrimos en entidades aisladas sean las mismas que operen en sistemas complejos y, además, las comunidades científicas que abordan los diferentes problemas manejan lenguajes diferentes, tienen sentidos e historias diversas. Por otra parte se encuentra la Teoría del Intercampo ${ }^{25}$ que identifica las relaciones existentes entre los fenómenos estudiados por disciplinas diferentes. Esta podría ser una alternativa interesante para la medicina, cuyo objeto de estudio -la persona enferma- es por definición transdisciplinario.

El concepto de «evidencia» debe ser separado de su legado positivista lógico, para incluir también lo que es evidente desde el contexto, la

\section{REFERENCIAS}

1. Hiтt J. Evidence-based medicine. New York Times Magazine. 2001.

2. JENICEK M. Epidemiology, evidence-based medicine, and evidence-based public health. J Epidemiol 1997; 7: 187-97. comunidad, y desde otras metodologías además de la experimental. La evidencia, así entendida, nos abre la posibilidad de mirar cómo el significado del lenguaje se modifica respecto de la posición del sujeto ${ }^{26}$. La gran oportunidad que nos otorga la polisemia del concepto, es recrearla desde abajo hacia arriba, explicitar las distintas suposiciones de práctica de investigación y aplicación clínica cotidiana y examinar los vínculos existentes entre los diversos niveles.

Esto implica una reorientación meta-teórica importante, generando, en primer lugar, un discurso científico en el cual los que lo utilizan, compartan las mismas suposiciones básicas sobre el tema que hablan. En términos prácticos, es la oportunidad para revisar también las prácticas y darles una coherencia conceptual que podría estar anclada en una mejor comprensión del objeto de estudio de la medicina, reconocer que las diversas aproximaciones de «conocer» contribuyen, y que las diversas metodologías son complementarias ${ }^{27,28}$.

La redefinición pluralista de sevidencia» implica sostener una perspectiva epistemológica en que un cuerpo de conocimientos se reconoce sólido y firme no por satisfacer determinados criterios axiomáticos, sino porque existe un entramado o red de relaciones coherentes, que otorga sentido y significado a ciertos hechos observables y los convierte en «evidencia»de algo. Esto es una lógica ecológica, que se acerca más a los postulados de una transdisciplina, o a un paradigma de la complejidad 29 .

Por lo tanto, en términos del científico-terapeuta el abordaje inicial interdisciplinario permitiría respetar la interacción entre las diversas dimensiones (objetos parciales) de estudio de las diversas disciplinas, e integrarlas en el objeto final de manera coherente, lógica y con sentido. Sentido final que es parte del objeto y del sujeto, en un campo perceptivo propio y que al mismo tiempo les precede y les sucede.

3. Davies H, Nutley S, Smith PC. What works? The role of evidence in public sector policy and practice. Public Money Management 1999; 19: 3-5.

4. FRIEDMan ST. Policies: science-based or scienceinformed? J Forest 2002; 100: 4. 
5. Gigerenzer G. Das Einmaleins der Skepsis: Über den richtigen Umgang mit Zahlen und Risiken. Berlin: Berliner Taschenbuch Verlag. 2004.

6. Louis P. Recherche sur les effets de la Signée. Paris: De Mignaret. 1835.

7. The Evidence-Based Medicine Working Group. Evidence-based medicine: a new approach to teaching the practice of medicine. JAMA 1992; 268: 2420-5.

8. Cochrane AL Effectiveness and efficiency. Random reflections on health services. Great Britain: Cambridge University Press 1971.

9. Рососк SJ. Clinical trials - a practical approach. Chichester: Wiley and Sons. 1983.

10. Wood M, FerLe E, Fitzgerald L. Achieving change in clinical practice: scientific, organizational and behavioural processes. Final report. Centre for Corporate Strategy and Change. Warwick Business School. The University of Warwick. 1998.

11. Ministerio de Salud de Chile. Reforma de Salud. Disponible en: www.minsal.cl/reforma [Consultado en septiembre 2004]

12. SACKETT DL Clinical epidemiology. What, who and whither. J Clin Epidemiol 2002; 55: 1161-6.

13. BеснтеL W. Philosophy of science. An overview for cognitive science. New Jersey: Lawrence Erlbaum Associates Publishers. 1988.

14. BeDREGAl P, FERLE E. Evidence based primary care? A multi-tier, multiple stakeholder perspective from Chile. Int J Health Plann Manage 2001; 16: 47-60.

15. Valkenburg G, Ashterhuis H, Nijhof A. Fundamental shortcomings of evidence-based medicine. J Health Organ Manage 2003; 17: 463-71.

16. PoPPER KR. La lógica de la investigación científica. Madrid: Tecnos. 1937/1971.
17. KuHN TS. La estructura de las revoluciones científicas. Chile: Fondo de Cultura Económica. 1971.

18. Feyerabend P. Tratado contra el método. Esquema de una teoría anarquista del conocimiento. Madrid: Tecnos. 1981.

19. FOUREZ G. La construcción del conocimiento científico. Sociología y ética de la Ciencia. Madrid: Narcea. 1998.

20. Quine W. Palabra y Objeto. Barcelona: Editorial Labor SA 1968; pag 88.

21. WHO. World Report on Knowledge for better health. WHO, Geneva. 2003.

22. WiNKLER F. Transferring power in health care. In: Health and Disease. A reader. Eds Davey B, Gray A and Seale C. Buckingham: Open University Press 1995.

23. SeEdhouse D. Ethics. The heart of health care. Second Edition. Chichester: Wiley. 1998.

24. Prigogine I. ¿El fin de la ciencia? Barcelona: Fried Schitman D. 1994.

25. Darden L, Maul N. Interfield theories. Philos Sci 1977; 44: 43-64.

26. Cornejo C. Who says what the words say? The problem of linguistic meaning in psychology. Theor Psychol 2004; 14: 5-28.

27. GoLDFELD M. Consensus in psychotherapy research and practice: Where have all the findings gone? Psychother Res 2000; 10: 1-16.

28. Косн S. The nature and limits of psychological knowledge. Lessons of a century qua «Science». Am Psychol 1981; 36: 257-69.

29. Morin E. Ciencia con conciencia. Barcelona: Anthropos. 1984. 